\title{
Gasto público en salud en Ecuador: ¿cumplimos con los compromisos internacionales?
}

\author{
Public Spending on Health in Ecuador: do we Comply \\ with International Commitments?
}
Gasto público em saúde no Equador: cumprimos com os compromissos internacionais?

\author{
Mauricio Guarderas \\ Universidad Andina Simón Bolívar, Sede Ecuador. Quito, Ecuador \\ mdguarderas@hotmail.com \\ http://orcid.org/0000-0002-4509-2362 \\ Diego Raza \\ Universidad Andina Simón Bolívar, Sede Ecuador. Quito, Ecuador \\ diego.raza@uasb.edu.ec \\ https://orcid.org/0000-0003-3258-6344

\section{Pamela González} \\ Universidad Andina Simón Bolívar, Sede Ecuador. Quito, Ecuador \\ pgonzales@udlanet.ec, pamegonzalezarcos90@hotmail.com \\ http://orcid.org/0000-0001-9205-4628
}

DOI: https://doi.org/10.32719/25506641.2021.9.10

Recibido: 2 de julio de 2020 • Revisado: 12 de julio de 2020 • Aceptado: 11 de agosto de 2020 Artículo de investigación

Licencia Creative Commons

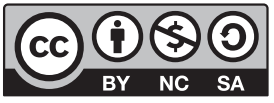




\section{Resumen}

Este estudio, de corte descriptivo, busca verificar si el gasto público en el presupuesto para la salud, entre 2008-2014, fue direccionado al cumplimiento de lo previsto en los marcos normativos supranacionales que buscan garantizar la atención de la salud como un derecho humano. A partir del análisis de los rubros de gasto de ese período, la metodología incluyó una categorización de cada ítem de gasto, en función del objetivo planteado en cada uno de los proyectos financiados, y su clasificación en las distintas dimensiones de salud que prevén los instrumentos normativos supranacionales de los que Ecuador es signatario. A partir de esa categorización y análisis se concluyó que el $95 \%$ de todos los recursos pudieron ser clasificados en alguna de las dimensiones previstas en los instrumentos internacionales y el $5 \%$ restante no correspondió a ninguna categoría, lo que significaría que aproximadamente USD 584 millones en ese período, fueron destinados a otros fines diferentes, dejando de ser enfocados en el fortalecimiento del sistema de salud pública de Ecuador que le permita garantizar el derecho a la salud de los ecuatorianos.

Palabras clave: presupuesto en salud, derecho a la salud, finanzas públicas, tratados internacionales, gasto público en salud.

JEL: I18 Salud, política de gobierno, regulación, salud pública.

\section{Abstract}

This descriptive study seeks to verify whether public spending on the health budget in the period 2008-2014 was directed to compliance with the provisions of supranational regulatory frameworks that seek to guarantee health care as a human right. Based on the analysis of that period's expenditure items, the methodology included a categorization of each item of expenditure, based on the objective set in each of the financed projects, and its classification in the different health dimensions provided by the supranational regulative instruments to which Ecuador is a signatory. Based on this categorization and analysis, it was concluded that $95 \%$ of all resources could be classified in any of the dimensions provided for international instruments and the remaining $5 \%$ did not correspond to any category, which would mean that approximately 584 million dollars were destined to other different purposes; ceasing to be focused on strengthening Ecuador's public health system that allows it to guarantee the Ecuadorian's right to health.

Keywords: Health budget, right to health, public finances, international treaties, public spending on health.

JEL: I18 Health, government policy, regulation, public health.

\section{Resumo}

Neste estudo, de recorte descritivo, procura-se verificar se o gasto público do orçamento para a saúde, entre os anos 2008 e 2014, foi direcionado ao cumprimento dos quadros normativos supranacionais que buscam garantir o atendimento de saúde como um direito humano. A partir da análise dos registros de gastos desse período, a metodologia incluiu uma categorização de cada item de gasto em função do objetivo estabelecido em 
cada um dos projetos financiados, além de sua classificação nas diferentes dimensões de saúde previstas nos instrumentos normativos supranacionais dos quais o Equador é signatário. Por meio dessa classificação e análise, concluiu-se que $95 \%$ de todos os recursos puderam ser classificados em alguma das dimensões previstas nos instrumentos internacionais, enquanto que os $5 \%$ restantes não corresponderam a nenhuma categoria, o que significaria que aproximadamente 584 milhões de dólares, durante tal período, foram destinados a outros fins, não sendo aplicados ao fortalecimento do sistema de saúde pública do Equador de forma a garantir o direito à saúde dos equatorianos.

Palavras-chave: Orçamento de saúde, direito à saúde, finanças públicas, tratados internacionais, gasto público em saúde.

JEL: I18 Saúde, política governamental, regulamentação, saúde pública.

$\mathrm{P}$ ara garantizar los derechos humanos, los gobiernos destinan recursos a los sectores sociales —salud, educación, entre otros-, en mayor o menor medida, dependiendo de las condiciones económicas prevalentes, el enfoque de política pública del gobierno de turno, y otros condicionantes.

Los recursos económicos de USD 10.700 millones destinados a precautelar la salud de todos los ecuatorianos durante el período 2008-2014, no se pueden tomar como única referencia para determinar si el Estado ha cumplido o no con su deber de precautelar la salud como un derecho de los ecuatorianos, ya que habrá que verificar cómo y en qué se gastaron esos valores. Los recursos destinados al sector salud deben ser evaluados en un contexto de procesos de larga duración y con una visión sostenible (Macías Sánchez et al. 2018), e incluso algunos autores afirman que ese gasto en salud debe evaluarse a la luz de la reducción de enfermedades o mejoras en la condición de vida de la población (Pinilla-Rodríguez, Jiménez-Aguilera y Montero-Granados 2018), de tal forma que esa relación de gasto público con mejoras de la calidad de vida, sirvan como insumo en la elaboración de políticas públicas (Lustig 2017).

La presente investigación, partiendo de la consideración de la salud como derecho humano, evalúa los recursos destinados a este sector durante el 2008 hasta el 2014, para verificar si han sido destinados al cumplimiento de normativas supranacionales que buscan garantizar dicho derecho. De manera complementaria, se busca estimar los recursos económicos que se gastaron y que no corresponden a la atención de los compromisos de cuidado de la salud, contenidos en dichos instrumentos supranacionales de los cuales Ecuador es signatario. 


\section{Revisión de la literatura}

\section{El rol del Estado en la salud pública}

La responsabilidad del Estado es precautelar el bienestar de los seres humanos, mejorando las condiciones que existen en su entorno, es decir, asignar recursos que aseguren los derechos básicos como la alimentación, educación, vivienda, salud, derecho al agua, entre otros, con la finalidad de proteger el derecho a la vida (Sen 2000).

El rol del Estado en la provisión de servicios, especialmente el servicio de salud pública, se puede comprender desde tres visiones diferentes que se resumen a continuación.

1. Liberal ortodoxa. De acuerdo con esta visión, la participación del Estado en el servicio de salud pública se circunscribe a crear condiciones en el mercado en el que intervengan actores privados para ofertar estos servicios a la población que, de su parte, tendrá una demanda natural derivada de su perfil epidemiológico. En esta visión, no hay elementos que contribuyan a considerar a la salud como un derecho y el Estado direcciona su responsabilidad a la libre competencia que existe entre mercados, mas no asegura este servicio como tal, limitándose al suministro de servicios altamente costo-efectivos, con el propósito de evitar externalidades negativas extremadamente nocivas para la sociedad (Navarro 2000).

Ya en la práctica, el servicio de salud en concordancia con este enfoque se apoya en el mercado de seguros, donde el usuario es valorado individualmente; también se evalúa el riesgo de cada beneficio de salud y, por otro lado, no se toma en cuenta factores socioculturales y demográficos como determinantes de la salud (Breilh 2013).

2. Liberal-redistributiva. Desde la perspectiva liberal-redistributiva, se plantea que el Estado debe impulsar una mirada universal, donde lo más importante sea mejorar las alternativas de vida de las personas mediante la creación de oportunidades relacionadas con la salud, así también como la educación, el trabajo, la vivienda, entre otros derechos elementales (Rawls 2009). Para ello, es necesario elaborar políticas públicas que involucren a los actores privados, con el propósito de trabajar en conjunto para el beneficio de la sociedad; por otro lado, es indispensable que cada individuo, y la co- 
munidad en su conjunto, sean responsables de su salud mejorando su estilo de vida para evitar factores que la afecten negativamente (Marmot 2003). En esta visión, se considera a la pobreza y la marginación como factores que causan enfermedades debido a la falta de servicios básicos necesarios para la subsistencia de cada ser humano.

En la práctica, el modelo liberal-redistributivo garantiza a toda la población el servicio universal de salud, es decir, todas las personas tienen acceso a esta prestación tomando en cuenta el nivel económico de cada individuo; en efecto, se promueve una relación Estado-mercado, esto quiere decir que las fuentes para subvencionar a la sociedad son los sistemas de impuesto, aportes de empleados y empleadores y gastos personales adicionales.

3. Marxista-igualitarista. Este modelo se basa en la prevención y la curación, tomando en cuenta la concepción de la enfermedad y su tratamiento. Entonces el concepto de salud se transforma en una serie de construcciones conjuntas, con cualidades de respeto a la diversificación, igualdad de derechos y oportunidades (Breilh 1986).

En la práctica, el Estado considera que la salud es un derecho humano prioritario y mejorará las condiciones de vida para que la sociedad disfrute de este servicio a plenitud según las necesidades que la población lo requiera (Borrero 2011).

El financiamiento de la salud se solventa por medio de impuestos que son dirigidos por el Estado a través de su presupuesto general. Con esto se genera libertad a los ciudadanos para que puedan acceder a servicios de salud gratuitos y con carácter de integralidad.

\section{La salud como derecho humano}

Según Stolkiner (2010) los derechos humanos a lo largo de la historia reflejan las perspectivas de los grupos de poder que los formulan en determinado momento y, por tanto, cambian de acuerdo a la manera en que las relaciones de poder evolucionan en la sociedad.

Se comprenden a los derechos humanos, además, como un propósito de interés colectivo que se exige como búsqueda de bienestar especialmente de las poblaciones menos favorecidas y con criterios de equidad, solidaridad y libertad (Villanueva Egan y Lezana Fernández 2016), que conduzcan al 
reconocimiento de la prevalencia del ser sobre las visiones economicistas de producción, rentabilidad, intercambiabilidad y objetivación de la dignidad humana (Appadurai 1991).

Ante esta concepción existen visiones antagónicas que pretenden limitar el concepto de la salud pretendiendo reducir a esquemas de libre mercado, frente a enfoques que buscan sistemas universales y gratuitos que garanticen el acceso a toda la población. Es de reconocer, sin embargo, que aun en las concepciones más extremas e inhumanas de liberalización radical y mercantilización de la salud, se comprende la necesidad de cuidar ciertos mínimos indeclinables o derechos humanos de baja intensidad (Santos 1998).

Para acercarse al concepto de salud, y al ejercicio específico que lo materializa como derecho, según Stolkiner (2010), se parte de ciertas contradicciones entre los aspectos objetivos y subjetivos que se encuentran presentes desde el vínculo de los profesionales y pacientes hasta el papel que cumplen los ciudadanos en la forma de recibir sus medicamentos y el servicio de cuidados hospitalarios. En este sentido, la discusión que se manifiesta en el panorama sobre la existencia del derecho a la salud tiene visiones exageradas, las cuales se oponen a este derecho, otras realizan un esquema reducido de justicia frente a un cuadro de libre mercado y sin tomar en cuenta las decisiones del Estado, puesto que actúa como un ente regulador, y otras perspectivas que determinan que el servicio de salud debe ser efectivamente considerado como un derecho humano $\mathrm{y}$, por tanto, ser gratuito.

En los servicios de salud se manifiestan excesos de mercado, lo cual se debería evitar a través de la gestión con justicia y equidad mediante el acercamiento universal a servicios sociales y promoviendo una gestión sostenible que perdure a largo plazo para el bienestar de la población. La equidad y la justicia se alcanzan mediante la financiación de servicios de salud de una manera directa a través de sistemas tributarios que garanticen una infraestructura sanitaria adecuada y de calidad para toda la población (Giraldo-Piedrahita 2015).

Para la materialización del derecho a la salud, es necesario crear programas, proyectos, presupuestos de capital que generen un incremento de bienestar en las personas que utilizan el servicio a la salud con la finalidad de prevenir y precautelar la vida de las personas, mediante el acceso universal a servicios que aseguren una vida digna. Esto se logrará si los recursos asig- 
nados para este rubro contemplan a todos los seres humanos, sin importar su clase social ni económica (Villanueva Egan y Lezana Fernández 2016).

\section{Revisión normativa}

\section{Marco normativo supranacional en salud pública}

El reconocimiento formal del derecho a la salud se da en el marco del Pacto Internacional de Derechos Económicos, Sociales y Culturales (Oficina del Alto Comisionado de las Naciones Unidas para los Derechos $\mathrm{Hu}-$ manos 1966) adoptado y abierto a la ratificación y adhesión de los Estados miembros de la Asamblea General de Naciones Unidas, mediante Resolución 2200, de 16 de diciembre de 1966 y que entró en vigor el 3 de enero de 1976, de acuerdo con el artículo 27 del propio instrumento. En este Pacto se reconoce el derecho a la salud en búsqueda de que todos los seres humanos gocen de bienestar físico y mental.

La consideración de la salud como un derecho debe considerar, entre otros factores, a la asistencia y atención médica, la prevención, curación y tratamiento para todas las personas - especialmente las más vulnerables-, así como el aseguramiento de que los niños y niñas de manera responsable reciban educación en sus hogares sobre higiene y sanidad.

El artículo 2 del Pacto demanda que sus países signatarios asignen todos los recursos que se establecen en la normativa local, con el fin de garantizar los derechos de las personas.

Por otra parte, en la Convención Americana sobre Derechos Humanos, suscrita en San José de Costa Rica, el 22 de noviembre de 1969 (OEA), en su artículo 26, indica que todos los Estados deben garantizar los derechos de la población a través de la asignación de su recursos y, en concordancia con la creación de políticas públicas que avalen este desarrollo.

Finalmente, el Protocolo adicional a la Convención Americana sobre Derechos Humanos en materia de Derechos Económicos, Sociales y Culturales, conocido como el Protocolo de San Salvador (OEA 1988), plantea las responsabilidades que debe cumplir el Estado frente a su pueblo: garantizar las condiciones de vida del pueblo y asegurar el desarrollo y el mejoramiento de 
factores sociales, culturales y económicos de cada familia para demostrar la protección del Estado hacia estas personas. El art. 10 de este Protocolo indica que el Estado reconoce como un bien público a la salud, y la importancia del aseguramiento de aspectos como la atención ambulatoria, es decir, de emergencia para todos los ciudadanos que necesitan el servicio, acceso universal a este derecho, prevención, curación y tratamiento para personas vulnerables a enfermedad y la priorización a personas consideradas de alto riesgo.

De forma complementaria, la Organización de las Naciones Unidas, a través de sus objetivos de desarrollo del milenio (ODM) de 2000 (Asamblea General de las Naciones Unidas 2000), definieron los objetivos sobre los derechos a la salud, planteados de la siguiente manera: reducir la mortalidad de los niños y niñas, desarrollar alternativas para mejorar la salud materna y, por último, combatir enfermedades catastróficas como el VIH, paludismo, entre otras. De la misma manera, los objetivos de desarrollo sostenible (ODS) tienen una nueva visión con respecto a la dirección de los Estados en el ámbito mundial, ya que los objetivos consisten en finalizar con la pobreza y proteger el medioambiente (Asamblea General de las Naciones Unidas 2015). Por otro lado, al momento de dirigir la atención al derecho a la salud, los ODS se enfocan en la priorización de los niños y niñas, por medio de la reducción de su índice de mortalidad, el desarrollo de nuevas técnicas que permitan el mejoramiento de la salud materna y buscar alternativas para combatir el VIH, malaria y otras enfermedades catastróficas para el ser humano. Si bien los ODM y ODS no tienen carácter normativo, muestran de manera complementaria la preocupación mundial por garantizar la salud como derecho humano.

Con estos antecedentes se puede concluir que los tres instrumentos normativos (de los cuales Ecuador es signatario) determinan las obligaciones del Estado y definen a la salud como un derecho.

\section{Orientaciones metodológicas}

Para la evaluación del cumplimiento del Estado ecuatoriano con los compromisos internacionales que devienen de su adhesión a los tres instrumentos reseñados en el marco normativo supranacional, se utilizaron las categorías y dimensiones que propone las Naciones Unidas (2008), para medir el cum- 
plimiento de los aspectos que determinan el derecho a la salud por parte de los países.

A continuación se presentan las categorías propuestas en dicho informe $\mathrm{y}$, en la columna contigua, sus correspondientes dimensiones del derecho a la salud:

\section{Tabla 1}

\section{Correspondencia entre las categorías propuestas por Naciones Unidas y las dimensiones del derecho a la salud}

\begin{tabular}{|l|l|}
\hline $\begin{array}{l}\text { Categorias propuestas por } \\
\text { Naciones Unidas }\end{array}$ & \multicolumn{1}{c|}{ Dimensiones del derecho a la salud } \\
\hline Atención a grupos vulnerables & Atención a personas con discapacidad. \\
\hline Salud mental & Salud mental. \\
\hline Medicamentos e insumos & Entrega de medicamentos esenciales y genéricos. \\
\hline $\begin{array}{l}\text { Promoción y prevención de la } \\
\text { salud }\end{array}$ & $\begin{array}{l}\text { Salud sexual y reproductiva, promoción de la salud, métodos } \\
\text { anticonceptivos, educación de niños y niñas en salud, chequeos } \\
\text { en edad escolar, vacunación, prevención del VIH, consumo de } \\
\text { drogas y alargamiento de la expectativa de vida. }\end{array}$ \\
\hline Salud materno infantil & $\begin{array}{l}\text { Aborto, salud y nutrición de la infancia, atención especializada } \\
\text { en el parto, cuidados prenatales, lactancia materna, mortalidad } \\
\text { prenatal y mortalidad materna. }\end{array}$ \\
\hline Tratamiento de enfermedades & $\begin{array}{l}\text { Tuberculosis, tratamiento de enfermedades transmisibles y no } \\
\text { transmisibles. }\end{array}$ \\
\hline Atención primaria de salud & Atención primaria de salud. \\
\hline Equipamiento e infraestructura & Equipamiento hospitalario, mejoramiento de infraestructura. \\
\hline Gobierno en salud & $\begin{array}{l}\text { Registro de nacimientos, muertes en sistemas de información, } \\
\text { investigaciones en salud, mejoramiento de capacidades, gobier- } \\
\text { no en salud en general. }\end{array}$ \\
\hline
\end{tabular}

Fuente: ONU (2018).

Elaboración propia.

A partir del análisis de los proyectos que ha llevado a cabo el Ministerio de Salud Pública del Ecuador (MSP) en el período estudiado, se busca identificar si los recursos empleados se han destinado a las mencionadas categorías y dimensiones previstas para evaluar el cumplimiento de la normativa internacional de derecho a la salud. 
La información de lo correspondiente al gasto del Gobierno ecuatoriano en salud dentro el período de análisis, se obtuvo de la página web del MSP, en la sección de transparencia/información financiera, que se publica periódicamente en el marco de la Ley Orgánica de Transparencia y Acceso a la Información Pública (LOTAIP).

Con estas consideraciones se identificaron los ítems de gasto del sector salud en el período analizado, se los clasificó en 91 proyectos de inversión y gasto corriente por cada año, y se los codificó con el número correspondiente a cada una de las nueve categorías del derecho a la salud, todo ello en función de los objetivos y componentes de cada uno de los proyectos.

La información desagregada del gasto en salud de la autoridad sanitaria ecuatoriana (gobierno central) para el período 2008-2014 está desplegada en una base de datos de 215.106 entradas. Cada una corresponde a un ítem de gasto específico atado a su vez a un proyecto de inversión o de gasto corriente con objetivos determinados. Dichos objetivos se alinean o no a una de las dimensiones del derecho a la salud establecidas en la metodología del Alto Comisionado de Derechos Humanos de las Naciones Unidas y el detalle de objetivos se encuentra dispobible en la página web del MSP, bajo la sección de transparencia institucional.

Para asegurar la adecuada categorización, se mantuvieron tres reuniones de trabajo con el coordinador general de Planificación del MSP en los meses de junio, octubre y noviembre de 2017, con el fin de validar que los objetivos y metas de los planes, programas y proyectos ejecutados en el período de análisis fueron adecuadamente interpretados y alineados a las dimensiones establecidas por la Oficina del Alto Comisionado de las Naciones Unidas para los Derechos Humanos (ACNUDH) para el derecho a la salud.

El siguiente paso fue realizar la sumatoria de los recursos asignados a cada dimensión agrupada del derecho a la salud, con la finalidad de conocer si el gasto público del período de análisis fue orientado correctamente al cumplimiento de los compromisos internacionales o simplemente se destinó para otros fines (categoría sin alineación). 


\section{Resultados}

Para iniciar el análisis, cabe indicar que la evolución del gasto público en salud para el período analizado creció del 1,57\% del PIB en 2008 al 2,17\% en el último año analizado, cifras correspondientes a un volumen de recursos de USD 968 millones para el primer año, que creció a USD 2210 millones para el último año, sumando un total de USD 10.700 millones para los 7 años analizados. Esto se puede observar en la tabla 2 en la que aparecen los valores de gasto público en salud para cada año del período analizado, y para cada una de las categorías de análisis.

Tabla 2

Gasto en las distintas categorías de atención a la salud como un derecho, en el período de análisis (millones de dólares)

\begin{tabular}{|c|c|c|c|c|c|c|c|c|}
\hline $\begin{array}{c}\text { Dimensión del } \\
\text { derecho a la } \\
\text { salud }\end{array}$ & 2008 & 2009 & 2010 & 2011 & 2012 & 2013 & 2014 & Total \\
\hline $\begin{array}{l}\text { Tratamiento de } \\
\text { enfermedades }\end{array}$ & 228,81 & 224,12 & 289,43 & 345,42 & 436,71 & 487,51 & 522,37 & 2534,37 \\
\hline $\begin{array}{l}\text { Equipamiento e } \\
\text { infraestructura }\end{array}$ & 204,79 & 200,60 & 259,05 & 309,16 & 390,87 & 436,33 & 467,54 & 2268,34 \\
\hline $\begin{array}{l}\text { Atención primaria } \\
\text { en salud }\end{array}$ & 104,55 & 102,40 & 132,25 & 157,83 & 199,54 & 222,75 & 238,68 & 1157,99 \\
\hline $\begin{array}{l}\text { Gobierno en } \\
\text { salud }\end{array}$ & 97,86 & 95,85 & 123,78 & 147,72 & 186,77 & 208,49 & 223,40 & 1083,87 \\
\hline $\begin{array}{l}\text { Medicamentos e } \\
\text { insumos }\end{array}$ & 95,73 & 93,77 & 121,10 & 144,52 & 182,72 & 203,97 & 218,56 & 1060,36 \\
\hline $\begin{array}{l}\text { Promoción de la } \\
\text { salud }\end{array}$ & 72,76 & 71,27 & 92,04 & 109,84 & 138,88 & 155,03 & 166,12 & 805,95 \\
\hline Sin alineación & 52,75 & 51,67 & 66,73 & 79,64 & 100,68 & 112,39 & 120,43 & 584,30 \\
\hline $\begin{array}{l}\text { Atención a grupos } \\
\text { vulnerables }\end{array}$ & 50,97 & 49,92 & 64,47 & 76,94 & 97,27 & 108,59 & 116,35 & 564,51 \\
\hline $\begin{array}{l}\text { Salud materno } \\
\text { infantil }\end{array}$ & 46,68 & 45,72 & 59,05 & 70,47 & 89,10 & 99,46 & 106,57 & 517,06 \\
\hline Salud mental & 13,29 & 13,02 & 16,81 & 20,07 & 25,37 & 28,32 & 30,35 & 147,23 \\
\hline Total & 968,20 & 948,35 & 1224,72 & 1461,60 & 1847,89 & 2062,85 & 2210,38 & $10.723,99$ \\
\hline
\end{tabular}

Fuente: MSP, página web en la sección de transparencia/información financiera (2008-2014).

Elaboración propia. 
Los recursos asignados que aparecen en la tabla 2 fueron distribuidos a 282 unidades ejecutoras en el ámbito nacional, esto quiere decir que el presupuesto fue repartido en hospitales y centros de atención en todo el territorio. De manera paralela, se ejecutaron 91 proyectos distribuidos en 23 programas. De todo el monto utilizado en el período de análisis, solo el $23 \%$ del total del gasto se utilizó como inversión y el $77 \%$ del gasto fue destinado como gasto corriente.

Luego del análisis se determinó que los recursos fueron destinados mayormente a la dimensión de tratamiento de enfermedades, lo que quiere decir que todavía en Ecuador la salud se enfoca más en la parte curativa que en la preventiva. Esta categoría mencionada anteriormente tuvo un gasto de USD 2534 millones, lo que representa el $24 \%$ del presupuesto; de este porcentaje, el $90 \%$ se destinó a los salarios para el personal de salud que trabajan para todos los establecimientos en el ámbito nacional.

En segundo lugar, los recursos fueron destinados a la dimensión de equipamiento e infraestructura, que recibió una cantidad de USD 2268 millones, que representan $21 \%$ del presupuesto; estos recursos se utilizaron para la construcción de 67 centros de salud, 10 nuevos hospitales y la repotenciación de varias unidades existentes.

En tercer lugar se ubica la atención primaria en salud; la cantidad asignada para este rubro fue de USD 1158 millones equivalente al $11 \%$ del presupuesto. De este porcentaje, el $93 \%$ fue utilizado para el pago de las remuneraciones del personal y el $7 \%$ restante para gastos operativos propios de cada unidad prestadora de los servicios.

La categoría gobierno en salud, que corresponde a los gastos relacionados con las dependencias administrativas del MSP en el ámbito nacional, manejó una cantidad de USD 1084 millones, que representa el $10 \%$ del total. De este porcentaje, el $73 \%$ fue para el pago de salarios del personal administrativo y el $27 \%$ restante, constituyeron gastos operativos de las unidades.

Para la dimensión de medicamentos e insumos se asignó una cantidad de USD 1060 millones que representó el $10 \%$ del total del presupuesto. Con estos datos, mencionados anteriormente, se puede concluir que el presupuesto en general fue efectivamente asignado para las diferentes categorías de salud propuestas por el ACNUDH. 
La sexta categoría referida a la promoción de la salud recibió la cantidad de USD 806 millones en el período analizado, equivalente al $8 \%$ del presupuesto.

Por otra parte, en la categoría denominada sin alineación se han ubicado todos los gastos que no tienen una vinculación con las dimensiones propuestas por la ACNUDH. La cantidad asignada para esta categoría fue de USD 584 millones y equivalen al $5 \%$ del presupuesto del período en estudio. No deja de alarmar que casi USD 600 millones aparentemente se hayan gastado en rubros que no necesariamente corresponden a lo que mandan los compromisos internacionales en materia de salud.

Siguiendo el análisis sobre el gasto público y su alineamiento con los compromisos de cumplimiento de normativa supranacional, en el período analizado se asignó una cantidad de USD 564 millones equivalentes al $5 \%$ a la dimensión de atención a grupos vulnerables. Por otro lado, se asignó una cantidad de USD 517 millones para los proyectos de salud materno infantil. Finalmente, la salud mental recibió una cantidad de USD 147 millones que representan el $1 \%$ del total del presupuesto.

Se debe alertar sobre los menores porcentajes de participación de estos rubros referidos a la atención de grupos vulnerables o de atención prioritaria, salud materno infantil y salud mental, que fueron destinatarios de menores cantidades de recursos que aquellas sin alineación que corresponden - como se ha dicho- a USD 584 millones en el período analizado, que se prefirió gastar en rubros que no corresponden a aquellos que deben atenderse para garantizar la atención de la salud como un derecho.

\section{Conclusiones}

Mediante la aplicación de la metodología de cálculo planteada, se evaluó de manera cualitativa el gasto público en salud del Ecuador en el período de análisis, cabe mencionar que esta metodología se basó en el cumplimiento de la normativa internacional descrita por las Naciones Unidas.

Luego de la aplicación de la metodología propuesta, se determinó que en general se cumplió con el objetivo de direccionar el gasto público al cumplimiento de la normativa internacional utilizada como referencia de estudio. 
Si bien los resultados permiten colegir que, en general, el gasto público se direccionó al cumplimiento de las normativas supranacionales, no se puede dejar de alertar sobre el hecho de que USD 584 millones que corresponde aproximadamente al $5 \%$ del total del presupuesto de esos años, se han agrupado en la categoría denominada sin alineación. Esto quiere decir que hay un $5 \%$ de gastos en ese período, que corresponde a más de USD 584 millones, y que según la información organizada corresponde a rubros como campañas publicitarias y de promoción institucional, varios contratos de servicios ocasionales, gastos de comisariato, impresiones, organización de eventos públicos y oficiales, entre otros. No se puede dejar de pensar que estos casi USD 600 millones pudieron haberse destinado mejor en proyectos e iniciativas que fortalezcan más directamente al sector salud, en previsión de una situación tan compleja de emergencia sanitaria como la que vive Ecuador al momento de escribir este artículo.

Este estudio y sus conclusiones tienen varias limitaciones que abren la puerta a futuras investigaciones que se podrían hacer para complementar el análisis.

Por una parte, el período de estudio es acotado a un número determinado de años. Por esta razón, en futuras investigaciones debería realizarse el mismo análisis para períodos anteriores y posteriores con el fin de contar con mayores elementos que puedan ratificar, corregir, o modular los resultados de esta investigación. Por otra parte, este estudio ha estado limitado a la indagación del cumplimiento de los tratados internacionales de los cuales Ecuador es signatario y que buscan garantizar el tratamiento de la salud como un derecho por parte del Estado. Esto debería complementarse en el futuro con la realización de otra investigación que verifique, en cambio, el cumplimiento con los mandatos normativos de carácter nacional, contenidos tanto en la Constitución de la República del Ecuador como su Ley Orgánica de Salud que, si bien tienen alta correspondencia - como es de esperar - con la normativa supranacional, constituyen un marco de referencia adicional que deberían examinarse en futuros estudios.

Finalmente, esta investigación debería dar lugar a futuros estudios en los que a partir de los resultados obtenidos, se busque identificar con otro tipo de metodologías, los impactos reales que ha generado el gasto público en salud ejecutado por el Gobierno ecuatoriano. 


\section{Referencias}

Appadurai, Arjun. 1991. La vida social de las cosas: perspectiva cultural de las mercancías. Ciudad de México: Grijalbo.

Borrero, Yadira. 2011. "Derecho a la salud, justicia sanitaria y globalización: un debate pendiente". Rev. Fac. Nac. Salud Pública 29 (3): 299-307. https://bit.ly/3hFiqSC.

Breilh, Jaime. 1986. Epidemiología: economía, medicina, y política. Ciudad de México: Fontamara.

---. 2013. "Addressing the Challenges of Social Determination of Health: A View from the South". Ponencia, University of Toronto, Dalla Lana School of Public Health. Accedido 28 de febrero. https://bit.ly/3iFjSFI.

EC. 2006. Ley Orgánica de Salud. Registro Oficial 298, Suplemento, 12 de octubre.

---. 2008. Constitución de la República del Ecuador. Registro Oficial 449, 20 de octubre.

Giraldo-Piedrahíta, Fernando. 2015. "Expresión de tres perspectivas de la justicia social en la organización de los sistemas de salud". Revista Gerencia y Políticas de Salud 14 (29): 27-40. https://bit.ly/3hBYPCH.

Guarderas Rivera, Mauricio David. 2019. “Análisis y evolución del gasto público en salud en el período 2008-2014". Tesis de maestría, Universidad Andina Simón Bolívar, Sede Ecuador, Quito. https://bit.ly/35IsLe4.

Lustig, Nora. 2017. "El impacto del sistema tributario y el gasto social en la distribución del ingreso y la pobreza en América Latina: Argentina, Bolivia, Brasil, Chile, Colombia, Costa Rica, Ecuador, El Salvador, Guatemala, Honduras, México, Nicaragua, Perú, República Dominicana, Uruguay y Venezuela. Una aplicación del marco metodológico del proyecto Compromiso con la Equidad (CEQ)". El trimestre económico 84 (335): 493-568. https:// bit.ly/2ZMyLP9.

Macías Sánchez, Alejandra, y Héctor Juan Villarreal Páez. 2018. "Sostenibilidad del gasto público: cobertura y financiamiento de enfermedades crónicas en México”. Ensayos. Revista de economía 37 (1): 99-133. https://bit.ly/3hD52hG.

Marmot, Michael G. 2003. "Understanding Social Inequalities in Health". Perspectives in Biology and Medicine 46 (3): 19-23. https://bit.ly/3c8LaSl.

Navarro, Vicente. 2000. The Political Economy of Social Inequalities: Consequences for Health and Quality of Life. Nueva York: Baywood Publishing Company.

OEA. 1969. Convención Americana sobre Derechos Humanos. Washington D. C.: OEA. https://bit.ly/35JBxsr.

---. 1988. Protocolo adicional a la Convención Americana sobre Derechos Humanos en materia de Derechos Económicos, Sociales y Culturales. Washington D. C.: OEA. https://bit. ly $/ 3 \mathrm{hEiwKj}$.

Oficina del Alto Comisionado de las Naciones Unidas para los Derechos Humanos. 1966. Pacto internacional de derechos económicos, sociales y culturales. Nueva York: ONU. https://bit.ly/3c5Ko8R. 
Organización de las Naciones Unidas (ONU). 2000. "Declaración del Milenio". Asamblea General de las Naciones Unidas. Nueva York: ONU. https://bit.ly/3c6wvHn.

---. 2008. Informe sobre los indicadores para promover y vigilar el ejercicio de los Derechos Humanos. Nueva York: ONU. https://bit.ly/2Rwc8di.

---. 2015. "Memoria del Secretario General sobre la labor de la Organización". Asamblea General de las Naciones Unidas. Nueva York: ONU. https://bit.ly/3muRLeQ.

Pinilla-Rodríguez, Diego Enrique, Juan de Dios Jiménez-Aguilera y Roberto Montero-Granados. 2018. "Gasto público y salud en el mundo, 1990-2012". Revista Cubana de Salud Pública 44 (2): 240-258. https://bit.ly/3iEWecr.

Rawls, John. 2009. A Theory of Justice. Cambridge: Harvard University Press.

Santos, Boaventura de Sousa. 1998. De la mano de Alicia: lo social y lo político en la postmodernidad. Bogotá: Siglo del Hombre Editores.

Sen, Amartya. 2000. Desarrollo y libertad. Buenos Aires: Planeta.

Stolkiner, Alicia. 2010. "Derechos humanos y derecho a la salud en América Latina: la doble faz de una idea potente". Medicina Social 5 (1): 89-95. https://bit.ly/2RBKKL3.

Villanueva Egan, Luis Alberto, y Miguel Ángel Lezana Fernández. 2016. "Derechos humanos y derecho a la salud: los desafíos contemporáneos”. Revista CONAMED 21 (4): 161-163. https://bit.ly/2H0FvSW. 